\section{Real-world outcomes and management strategies for venetoclax-treated chronic lymphocytic leukemia patients in the United States}

\author{
Anthony R. Mato, ${ }^{1}$ Meghan Thompson, ${ }^{2}$ John N. Allan, ${ }^{3}$ Danielle M. Brander, ${ }^{4}$ \\ John M. Pagel, ${ }^{5}$ Chaitra S. Ujjani, ${ }^{6}$ Brian T. Hill, ${ }^{7}$ Nicole Lamanna, ${ }^{8}$ \\ Frederick Lansigan, ${ }^{9}$ Ryan Jacobs, ${ }^{10}$ Mazyar Shadman, ${ }^{11}$ Alan P. Skarbnik, ${ }^{12}$ \\ Jeffrey J. Pu, ${ }^{13}$ Paul M. Barr, ${ }^{14}$ Alison R. Sehgal, ${ }^{15}$ Bruce D. Cheson, ${ }^{6}$ \\ Clive S. Zent, ${ }^{14}$ Hande H. Tuncer, ${ }^{16}$ Stephen J. Schuster, ${ }^{2}$ Peter V. Pickens, ${ }^{17}$ \\ Nirav N. Shah, ${ }^{18}$ Andre Goy, ${ }^{12}$ Allison M. Winter, ${ }^{7}$ Christine Garcia,${ }^{15}$ \\ Kaitlin Kennard, ${ }^{2}$ Krista Isaac, ${ }^{19}$ Colleen Dorsey, ${ }^{2}$ Lisa M. Gashonia, ${ }^{2}$ \\ Arun K. Singavi, ${ }^{18}$ Lindsey E. Roeker, ${ }^{1}$ Andrew Zelenetz, ${ }^{1}$ Annalynn Williams, ${ }^{14}$ \\ Christina Howlett, ${ }^{12}$ Hanna Weissbrot, ${ }^{8}$ Naveed Ali, ${ }^{17}$ Sirin Khajavian, ${ }^{11}$ \\ Andrea Sitlinger, ${ }^{4}$ Eve Tranchito, ${ }^{7}$ Joanna Rhodes, ${ }^{2}$ Joshua Felsenfeld, ${ }^{3}$ \\ Neil Bailey, ${ }^{5}$ Bhavisha Patel, ${ }^{20}$ Timothy F. Burns, ${ }^{9}$ Melissa Yacur, ${ }^{13}$ \\ Mansi Malhotra, ${ }^{16}$ Jakub Svoboda, $^{2}$ Richard R. Furman ${ }^{3}$ and Chadi Nabhan ${ }^{21}$ \\ ARM and MT contributed equally to this work.
}

${ }^{1}$ CLL Program, Leukemia Service, Division of Hematologic Oncology, Department of Internal Medicine, Memorial Sloan Kettering Cancer Center, New York, NY; ${ }^{2}$ Center for CLL, Division of Hematology and Oncology, University of Pennsylvania, Philadelphia, PA; ${ }^{3}$ New York Presbyterian \& Weill Cornell, NY; ${ }^{4}$ Division of Hematologic Malignancies and Cellular Therapy, Duke University, Durham, NC; ${ }^{5}$ Center for Blood Disorders and Stem Cell Transplantation, Swedish Cancer Institute, Seattle, WA; ${ }^{6}$ Georgetown University Hospital Lombardi Comprehensive Cancer Center, Washington, DC; ${ }^{7} T a u s s i g$ Cancer Institute, Cleveland Clinic Foundation, $\mathrm{OH} ;{ }^{8} \mathrm{Columbia}$ University Medical Center, New York, NY; ${ }^{9}$ Dartmouth-Hitchcock Medical Center, Lebanon, NH; ${ }^{10}$ Department of Hematologic Oncology and Blood Disorders, Levine Cancer Institute, Carolinas Healthcare System, Charlotte, NC; ${ }^{11}$ University of Washington/Fred Hutchinson Cancer Research Center, Seattle Cancer Care Alliance, WA; ${ }^{12}$ John Theurer Cancer Center, Hackensack Meridian Health, NJ; ${ }^{13}$ Penn State Health, Hershey, PA; ${ }^{14}$ Wilmot Cancer Institute Division of Hematology/Oncology, University of Rochester Medical Center, NY; ${ }^{15}$ University of Pittsburgh Medical Center, PA; ${ }^{16}$ Tufts Medical Center, Boston, MA; ${ }^{17}$ Abington Hem. Onc. Assoc., Inc., Willow Grove, PA; ${ }^{18}$ Division of Hematology \& Oncology, Medical College of Wisconsin, Brookfield, WI; ${ }^{19}$ Internal Medicine, Lankenau Medical Center, Wynnewood, PA; ${ }^{20}$ Washington Hospital Center, DC and ${ }^{21}$ Cardinal Health, Dublin, OH, USA

\section{ABSTRACT}

$\mathrm{V}$ enetoclax is a BCL2 inhibitor approved for $17 p$-deleted relapsed/refractory chronic lymphocytic leukemia with activity following kinase inhibitors. We conducted a multicenter retrospective cohort analysis of patients with chronic lymphocytic leukemia treated with venetoclax to describe outcomes, toxicities, and treatment selection following venetoclax discontinuation. A total of 141 chronic lymphocytic leukemia patients were included ( $98 \%$ relapsed/refractory). Median age at venetoclax initiation was 67 years (range 37-91), median prior therapies was $3(0-11), 81 \%$ unmutated $I G H V, 45 \% \operatorname{del}(17 p)$, and $26.8 \%$ complex karyotype ( $\geq 3$ abnormalities). Prior to venetoclax initiation, $89 \%$ received a B-cell receptor antagonist. For tumor lysis syndrome prophylaxis, 93\% received allopurinol, 92\% normal saline, and $45 \%$ rasburicase. Dose escalation to the maximum recommended dose of $400 \mathrm{mg}$ daily was achieved in $85 \%$ of patients. Adverse events of interest included neutropenia in $47.4 \%$, thrombocytopenia in 36\%, tumor lysis syndrome in $13.4 \%$, neutropenic fever in $11.6 \%$, and diarrhea in $7.3 \%$. The overall response rate to venetoclax was $72 \%(19.4 \%$ complete remission). With a median follow up of 7 months, median progression free survival and overall survival for the entire cohort have not been reached. To date, 41 venetoclax treated patients have discontinued

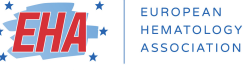

Haematologica 2018

Volume 103(9):1511-1517

\author{
Correspondence: \\ matoa@mskcc.org \\ Received: March 17, 2018. \\ Accepted: June 5, 2018. \\ Pre-published: June 7, 2018.
}

doi:10.3324/haematol.2018.193615

Check the online version for the most updated information on this article, online supplements, and information on authorship \& disclosures: www.haematologica.org/content/103/9/1511

(C)2018 Ferrata Storti Foundation

Material published in Haematologica is covered by copyright. All rights are reserved to the Ferrata Storti Foundation. Use of published material is allowed under the following terms and conditions:

https://creativecommons.org/licenses/by-nc/4.0/legalcode. Copies of published material are allowed for personal or internal use. Sharing published material for non-commercial purposes is subject to the following conditions:

https://creativecommons.org/licenses/by-nc/4.0/legalcode, sect. 3. Reproducing and sharing published material for commercial purposes is not allowed without permission in writing from the publisher. 
therapy and 24 have received a subsequent therapy, most commonly ibrutinib. In the largest clinical experience of venetoclax-treated chronic lymphocytic leukemia patients, the majority successfully completed and maintained a maximum recommended dose. Response rates and duration of response appear comparable to clinical trial data. Venetoclax was active in patients with mutations known to confer ibrutinib resistance. Optimal sequencing of newer chronic lymphocytic leukemia therapies requires further study.

\section{Introduction}

Venetoclax is an oral second-generation BCL2 inhibitor with demonstrated activity and durable responses in relapsed/refractory (R/R) chronic lymphocytic leukemia (CLL), including those with chromosome 17p deletion (del(17p)), unmutated IGHV, fludarabine-resistance, bulky disease, and progression on or following ibrutinib or idelalisib. ${ }^{1-5}$ Overall response rate (ORR) to venetoclax monotherapy was $79 \%$ in the phase II trial in $\operatorname{del}(17 p) R / R$ CLL. ${ }^{3}$ Moreover, ORRs were $65 \%$ and $67 \%$ in R/R CLL following ibrutinib or idelalisib, respectively. ${ }^{4,5}$ In addition to data with monotherapy, progression free survival (PFS) was superior for patients treated with venetoclax and rituximab compared to bendamustine and rituximab in $\mathrm{R} / \mathrm{R}$ CLL, and more venetoclax-treated patients achieved undetectable minimal residual disease (MRD) $(83.5 \%$ vs. $23.1 \%)^{6}$

Knowledge about venetoclax efficacy, dose-escalation, and toxicity in CLL patients has almost entirely been informed by experiences from clinical trials. ${ }^{3}$ As several real-world evidence series showed that toxicity profiles and outcomes for kinase inhibitor-treated patients may differ from those reported in the clinical trial setting, studying whether these differences apply to venetoclaxtreated patients is essential. ${ }^{7-11}$ Furthermore, there is one study to date regarding strategies for early identification of high-risk patients, particularly those previously treated with kinase inhibitor based therapy, for progression on venetoclax and how treatment is selected after its discontinuation is selected. ${ }^{2}$

We aimed to better understand disease characteristics and toxicities of CLL patients treated with venetoclax in clinical practice and contrast their outcomes to those reported in key clinical trials. We explored prognostic factors that predict early progression on venetoclax and studied treatment selection following discontinuation. To our knowledge, this analysis reports the largest series of CLL patients treated with venetoclax in a real-world setting with a focus on outcomes following venetoclax discontinuation.

\section{Methods}

We conducted a multicenter, retrospective cohort study of all CLL patients treated with venetoclax across 19 United States academic and community cancer centers. The study was approved by the institutional review board at each US institution. Investigators conducted a detailed review of the institutional electronic medical records to identify patients with CLL treated with venetoclax. Collected data included demographics, clinical and genetic prognostic factors, venetoclax dose-escalation management, long-term dosing, toxicities, tumor lysis syndrome (TLS) prophylaxis strategies and outcomes, ORR, complete response (CR), survival out- comes, and reasons for discontinuation. Investigators were asked to follow the National Cancer Institute working group international workshop guidelines for CLL (iwCLL) published in 2008 to define rates of response and progression of disease. ${ }^{12}$ Disease burden as a predictor for TLS risk was categorized as low, medium, and high per the treating physician. Physicians were asked to use the venetoclax package insert to guide the categorization, which was developed based on United States approval of the drug in 2016. ${ }^{13}$ TLS events were defined as per Howard criteria, which specify criteria for laboratory and clinical TLS. ${ }^{14}$ Adverse events (AEs) were graded using the NCI Common Toxicity Terminology Criteria for Adverse Events v4.0 (CTCAE v4.0). Cytogenetics, FISH results and next generation sequencing (NGS) were reported for patients where available.

The primary endpoint was PFS, defined as the time from venetoclax initiation until progression or death from any cause as per the Kaplan Meier method. ${ }^{15}$ Patients were censored at the time of last follow up or at the time of next therapy regardless of progression status. Outcomes were stratified by prognostic characteristics where available, including del(17p) status, complex karyotype (>3 abnormalities), and venetoclax monotherapy versus combinations. Secondary endpoints included overall survival (OS), venetoclax dosing and toxicities, TLS incidence, dose escalation schema, response rates, and reasons for discontinuation.

Comparisons of survival outcomes data were made using the long rank (LR) test. ${ }^{16}$ Hazard ratios were estimated using Cox regression analyses. ${ }^{17}$ Other analyses were descriptive. Tests were two-sided at the $5 \%$ level. Statistical analyses were performed using STATA 10.1 (Stata Statistical Software: Release 10, 2007; StataCorp LP, College Station, TX).

\section{Results}

\section{Patient characteristics}

We identified 141 CLL patients treated with venetoclax. Males and Caucasians represented most patients at $66 \%$ and $87 \%$, respectively. The median age at diagnosis was 59 years (range $30-88$ ), and median age at venetoclax initiation was 67 years (range 37-91). The population consisted almost entirely of patients with R/R CLL, with only 2 $(1.4 \%$ of 141$)$ of patients being treatment-naive. Patients had received a median of 3 prior therapies (range $0-11$ ). Venetoclax was administered in combinations in $18.4 \%$ ( $n=26$ of 141 ) of patients. Ibrutinib (36\%), obinutuzumab $(32 \%)$, and rituximab (24\%) were the most commonly used drugs with venetoclax. Almost $89 \%$ of patients were treated with a B-cell receptor signal transduction inhibitor prior to venetoclax; $82 \%(n=115 / 141)$ received ibrutinib. Patient characteristics are summarized in Table 1.

Most patients in this cohort had at least one traditionally poor-risk feature: $45 \%$ ( $n=61 / 136$ tested) patients had chromosome del(17p), 26\% (n=34/131) had deletion of chromosome 11q (del(11q)), 44\% (n=42/95) had p53 mutations, $26.8 \%(n=52 / 130)$ had a complex karyotype 
( $>3$ chromosomal abnormalities) and $26.8 \%(n=15 / 56)$ had a NOTCH1 mutation. Thirty-one patients $(22 \%)$ had del(17p) and TP53 mutation, and 16 patients (11\%) had del(17p), TP53 mutation, and complex karyotype.

\section{Venetoclax dosing and adverse events}

All patients underwent 5-week dose escalation of venetoclax. During dose-escalation, $85 \% \quad(n=120 / 141)$ of patients achieved a maximum dose of $400 \mathrm{mg}$ daily, with $75 \%$ of the cohort ( $\mathrm{n}=103 / 137)$ maintaining $400 \mathrm{mg}$ daily as a long-term stable dose. Dose interruptions occurred in $30 \%$ of patients and $21 \%$ required dose reduction. Details are available in Online Supplementary Table S1. Reasons for interruptions were not available. Hematologic events were the most common AEs with neutropenia (defined as ANC $<1000$ cells/microL) occurring in almost half of patients (47.4\%, $\mathrm{n}=65 / 137)$ and thrombocytopenia (defined as platelets $<50,000$ cells/microL) occurring in over one-third of patients $(36.0 \%, n=49 / 136)$. Other AEs included TLS $(13.4 \%, \mathrm{n}=18 / 134)$, neutropenic fever $(11.6 \%, \mathrm{n}=16 / 138)$, and grade $\geq 2$ diarrhea $(7.3 \%$, $n=10 / 138)$. Opportunistic infections (OI) while on venetoclax were reported in 11 patients $(7.8 \%)$ with the three most common being pneumocystis jirovecii pneumonia (PJP) $(n=6)$, invasive fungal $(n=2)$, and toxoplasmosis $(\mathrm{n}=2)$. Nine OI events occurred in patients with prior exposure to any kinase inhibitor, 8 OI events occurred in patients with prior exposure to ibrutinib, and 6 OI events occurred in patients with prior exposure to two prior kinase inhibitors. The median time from venetoclax start to OI event was 5 months ( $0.2-16$ months).

\section{TLS: prophylaxis and hospitalization practice patterns}

Of 134 patients with TLS data, $44.8 \%$ were low-risk $(n=60), 35.8 \%$ were intermediate risk $(n=48)$, and $19.4 \%$ were high risk $(\mathrm{n}=26)$. Eighty-nine of 131 patients $(64.3 \%)$ had pre-venetoclax lymph node assessment by CT scan to inform TLS risk. To minimize the occurrence of TLS, allopurinol was used in almost all cases regardless of risk category $(93.1 \%$ low risk, $87.5 \%$ intermediate risk, $100.0 \%$ for high risk), as was intravenous normal saline (82.1\% low risk, $91.7 \%$ intermediate risk, $100.0 \%$ high risk). Rasburicase use as TLS prophylaxis varied by risk category: $17.2 \%$ of low risk, $31.3 \%$ of intermediate risk, and $46.2 \%$ of high risk patients. TLS risk stratification, prophylaxis patterns, and incidence are summarized in Table 2.

Most patients had one or more planned hospitalizations during dose escalation regardless of risk category (Online Supplemental Table S2). Twenty-seven patients were not hospitalized at any point, including 20 of $58(34.5 \%)$ low risk patients and 7 of $48(14.6 \%)$ intermediate risk patients. All high-risk patients were hospitalized at least once for TLS monitoring and prophylaxis during the dose escalation phase. Among high-risk patients, $32.0 \%$ ( $n=8$ of 25) were hospitalized for all five dose escalations. The mean number of days hospitalized during the 5-week dose escalation period for low risk, intermediate risk, and high-risk patients were 1.5, 1.7, and 3.1, respectively.

Overall, the incidence of TLS events (laboratory and clinical) was $13.4 \%(\mathrm{n}=18 / 134)$ with 5 events $(3.7 \%)$ reported in low-risk, 4 (3.0\%) in intermediate-risk, and 9 $(6.7 \%)$ in high-risk patients. Of these events, 6 were recorded as clinical TLS events (2 low risk patients, 1 intermediate risk patient, and 3 high risk patients), and the
Table 1. Baseline characteristics of 141 patients treated with venetoclax.

\begin{tabular}{lc}
\hline Patient characteristics & Median (range) \\
Median age at diagnosis, years & $59(30-88)$ \\
Median age at venetoclax start, years & $67(37-91)$ \\
\hline Median prior lines of therapy & $3(0-11)$ \\
Follow up, months* & $7(0.1-38.4)$ \\
\hline CLL characteristics & Frequency \\
& (n with characteristic/ \\
& total $n$ with available data)
\end{tabular}

Relapsed/Refractory

i $98.6 \%(139 / 141)$

Treatment naive

$1.4 \%(2 / 141)$

CLL genetics

$\operatorname{Del}(17 p)$

$44.9 \%(61 / 136)$

$\operatorname{Del}(11 q)$

$26.0 \%(34 / 131)$

TP53 mutation

$44.2 \%(42 / 95)$

NOTCH1 mutation

$26.8 \%(15 / 56)$

Complex karyotype, $\geq 3$ mutations

$26.8 \%(52 / 130)$

Unmutated IGHV

$83.3 \%(60 / 72)$

Prior ibrutinib exposure

$81.6 \%(115 / 141)$

Ibrutinib resistance mutations

BTK mutation $\quad 35.3 \%(12 / 34)$

PLC 2 mutation

$12.5 \%(4 / 32)$

Venetoclax administered in combination

$18.4 \%(26 / 141)$

Venetoclax and ibrutinib

$36 \%(9 / 26)$

Venetoclax and obinutuzumab

$32 \%(8 / 26)$

Venetoclax and rituximab

$24 \%(6 / 26)$

*Median follow up calculated using overall survival.

remainder were laboratory events $(n=12)$. Of the clinical TLS patients, 4 of 6 achieved $400 \mathrm{mg}$ venetoclax dosing. No TLS patient required hemodialysis. One TLS death was reported in a patient who was re-challenged with venetoclax after a delayed interruption without utilizing a dose escalation schedule or hospitalization for venetoclax re-escalation. We were unable to correlate TLS events with a threshold dose of venetoclax.

\section{Outcomes}

The reported ORR and CR rate, stratified by selected risk factors, are summarized in Table 3. The ORR for the entire cohort was $72.1 \%$ and $19.4 \%$ of patients achieved a CR. The median time to best response was 2.1 months. Venetoclax had a similar ORR across several high-risk groups including patients with age $\geq 65(\mathrm{ORR}=74.3 \%)$, $\operatorname{del}(17 p)(71.4 \%)$, prior ibrutinib therapy $(69.1 \%)$, BTK mutation $(91.6 \%)$, and PLC 2 mutation $(75.0 \%)$. At a median follow up of 7 months, the median PFS and OS have not been reached for the entire cohort (Figure 1a and 1b). The projected PFS and OS for the entire cohort at 12 months were $68 \%$ and $88 \%$, respectively. Patients with a TP53 interruption (del(17p) and/or TP53 mutation) had significantly shorter PFS than those with intact TP53 (Figure 1c), though OS for the two groups was not significantly different (Online Supplementary Figure S1).

In univariate analyses, we identified TP53 interruption as a predictor of inferior PFS (HR 2.7, 95\% CI 1.08-6.7, $P=0.034$ ) but not OS (HR 1.78, 95\% CI .55-5.74, $P=.332$ ). 
Complex karyotype (HR 1.36, 95\% CI 0.66-2.84, $P=0.4$ ), prior ibrutinib therapy $(1.74,95 \% \mathrm{CI} 0.61-5.0, P=0.3)$, and unmutated IGHV (HR $0.29,95 \%$ CI $0.04-2.3, P=0.25$ ) were not significantly associated with inferior PFS. TP53 interruption remained a significant predictor for inferior PFS in a multivariate analyses which included TP53 interruption, complex karyotype and prior ibrutinib therapy (HR 2.8, CI 1.22-6.4, $P=0.03$ ). The presence of del(11q) did not impact OS and had an observed protective effect on PFS (HR 0.31, 95\% CI 0.11-0.90, $P=0.03$ ).

\section{Venetoclax discontinuations and treatment selection following venetoclax}

Venetoclax was discontinued in 41 patients (29\%). Progression of disease was the most common reason for discontinuation $(53.8 \%, \mathrm{n}=21)$ followed by toxicity $(20.5 \%, n=9)$, two-thirds of which were hematologic. Other reasons for discontinuation included death not related to progressive disease $(10.25 \%, \mathrm{n}=4)$, second cancer $(5.1 \%, \mathrm{n}=2)$, physician/patient preference $(2.5 \%, \mathrm{n}=1)$, Richter's transformation $(2.5 \%, \mathrm{n}=1)$, and planned alternate therapy including CD19 directed chimeric antigen receptor $\mathrm{T}$ cells (CAR-T, $2.5 \%, \mathrm{n}=1$ ) and transplantation $(2.5 \%, \mathrm{n}=1)$.

Table 4 summarizes therapy selection and outcomes for individual cases following venetoclax. Notably, 17 of 34 patients $(50 \%)$ who discontinued venetoclax and remain alive have not required a subsequent therapy. Reasons for discontinuation in the group of patients who have not yet been treated following venetoclax discontinuation include toxicity $(n=6)$, progression of CLL $(n=4)$, death not secondary to toxicity or progression $(n=4)$, secondary malignancy $(n=2)$, and doctor or patient preference $(n=1)$. Ibrutinib-based therapy was the most common choice after venetoclax; five of $24(21 \%)$ patients receiving ibrutinib. Three of five of patients treated with ibrutinib had prior ibrutinib exposure. Of these five patients, 1 had a partial response, 2 had stable disease, and 2 had progressive disease. Other therapies selected included rituximab monotherapy $(12.5 \%, \mathrm{n}=3)$, anthracycline based regimens $(12.5 \%, \mathrm{n}=3)$, allogeneic stem cell transplant $(12.5 \%, n=3)$, idelalisib-based therapy $(8.3 \%, n=2)$, and CAR-T $(8.3 \%, n=2)$. Subsequent lines of therapies with their corresponding responses are detailed in Online Supplementary Table S3.

\section{Discussion}

In the largest series of venetoclax-treated CLL patients treated in the U.S., response rates (ORR 72.1\%) and survival data are comparable to those reported in published clinical trials. ${ }^{1,3-5}$ Toxicities were similar with hematologic toxicities being the most frequently observed. Rates of TLS were higher than prior reports. Collectively, these results suggest that the efficacy and safety profile of venetoclax demonstrated in the clinical trials setting are comparable to what has been observed in the real world.

Consistent with previously published data, the ORR for the del $(17 p)$ population remained high at $71.4 \%$. Whereas

Table 2. Tumor lysis syndrome prophylaxis and events.

\begin{tabular}{|c|c|c|c|c|c|c|}
\hline $\begin{array}{l}\text { ILS risk category } \\
n=134\end{array}$ & Allopurinol & $\begin{array}{l}\text { TLS prophylaxis } \\
\text { Rasburicase }\end{array}$ & Normal saline & Total & $\begin{array}{l}\text { TLS events } \\
\text { Laboratory }\end{array}$ & Clinical \\
\hline $\begin{array}{l}\text { Low } \\
44.8 \%(\mathrm{n}=60)\end{array}$ & $\begin{array}{c}93.1 \% \\
(\mathrm{n}=54 / 58)\end{array}$ & $\begin{array}{c}17.2 \% \\
(\mathrm{n}=10 / 58)\end{array}$ & $\begin{array}{c}82.1 \% \\
(\mathrm{n}=46 / 56)\end{array}$ & 5 & 3 & 2 \\
\hline $\begin{array}{l}\text { Intermediate } \\
35.8 \%(\mathrm{n}=48)\end{array}$ & $\begin{array}{c}87.5 \% \\
(\mathrm{n}=42 / 48)\end{array}$ & $\begin{array}{c}31.3 \% \\
(\mathrm{n}=15 / 48)\end{array}$ & $\begin{array}{c}91.7 \% \\
(\mathrm{n}=44 / 48)\end{array}$ & 4 & 3 & 1 \\
\hline $\begin{array}{l}\text { High } \\
19.4 \%(\mathrm{n}=26)\end{array}$ & $\begin{array}{c}100.0 \% \\
(\mathrm{n}=26 / 26)\end{array}$ & $\begin{array}{c}46.2 \% \\
(\mathrm{n}=11 / 26)\end{array}$ & $\begin{array}{c}100.0 \% \\
(\mathrm{n}=25 / 25)\end{array}$ & 9 & 6 & 3 \\
\hline
\end{tabular}

A

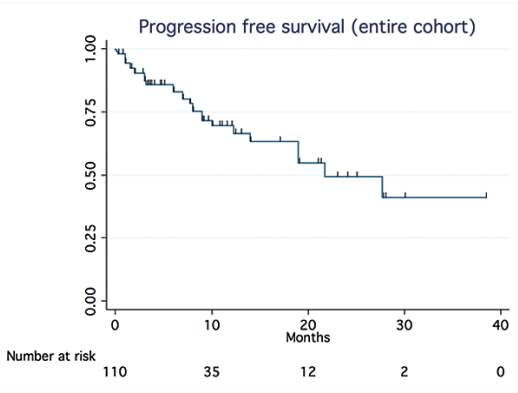

B

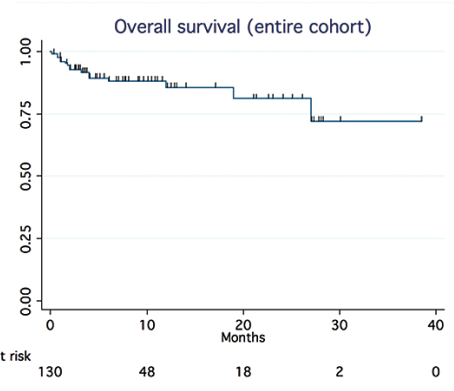

C

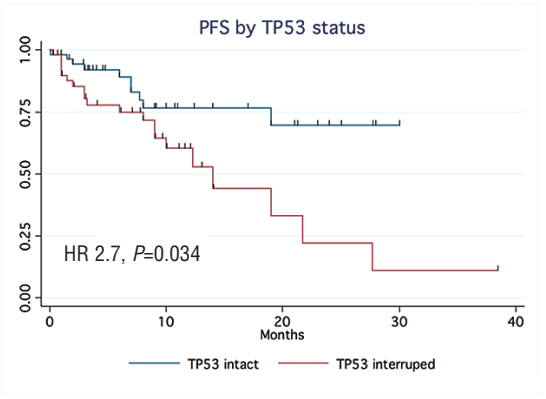

Figure 1. Survival analyses for patients following venetoclax initiation. (A) Progression free survival for the entire cohort. Median PFS has not been reached with median follow up of 7 months. Projected 12-month PFS is 68\%. (B) Overall survival for the entire cohort. Median OS has not been reached with median follow up of 7 months. Projected 12 -month OS is $88 \%$. (C) Progression free survival by TP53 status. PFS is significantly superior for patients with intact TP53 compared to patients with TP53 interruption, either TP53 mutation or del(17p). 
TP53 interruption was significantly associated with inferior PFS, OS was not compromised. Complex karyotype was not associated with inferior PFS, despite being shown to be a risk factor for progression in patients receiving venetoclax in a recent study by Anderson et al. ${ }^{2}$ It is possible that our shorter follow up accounts for this discrepancy. Interestingly, del(11q) did not adversely affect PFS. Similar findings were reported by Kipps et al. in 620 patients treated with ibrutinib stratified by del(11q) status (HR 0.73, $P=0.08$ ). ${ }^{18}$ In subgroup analysis, the response rate for patients previously treated with ibrutinib was $69.1 \%$, similar to the $65 \%$ demonstrated by Jones et al. in patients who were treated with venetoclax following ibrutinib. ${ }^{5}$ Finally, we did not observe a difference in PFS whether venetoclax was given alone or in combination.

TLS has been suggested as the most critical toxicity with venetoclax, contributing to early treatment-related deaths in clinical trials, particularly before the current dose rampup schedule was implemented in trials to minimize TLS risk. In our study, TLS rates were higher than those reported in most recent trials. In this cohort, 18 patients $(13.4 \%)$ had TLS; $12(9.0 \%)$ cases were laboratory TLS events and $6(4.5 \%)$ cases were clinical events. In the initial phase I venetoclax study, $18 \%$ of patients experienced TLS (12.5\% laboratory, $5.6 \%$ clinical). However, once the dosing schedule was modified to minimize TLS risk, $1.7 \%$ of patients had laboratory TLS and none had clinical TLS. ${ }^{3}$ More recently reported clinical trials using the standard dose ramp-up protocol have shown laboratory TLS rates of $2.2 \%{ }^{5}$ and $4.7 \%^{3}$ and clinical TLS rates of $0 \%{ }^{3.5}$ Despite the higher rates of TLS observed in our study, only $19.4 \%$ of patients were deemed high risk for TLS versus $25-49 \%$ of patients classified as high risk in the clinical trials setting. ${ }^{3.5}$

Overall, adherence to TLS prophylaxis recommendations was excellent. The majority (92\%) received allopurinol, which is recommended for all patients regardless of risk category ${ }^{13}$ Guidelines per the US venetoclax prescribing information document also recommend oral hydration and intravenous normal saline for high risk patients, which was followed for all patients in our cohort. Similarly, rasburicase is recommended for patients with elevated baseline uric acid. This was used in over onequarter of all cases and almost half of the high-risk cases. All high-risk patients had at least one planned hospitalization during dose escalation. Three of 25 high-risk patients forwent the second recommended hospitalization given lack of TLS development during the first hospitalization.
Most low and intermediate-risk patients were hospitalized at least once, suggesting a conservative approach was utilized in this series during the dose ramp-up as outpatient dosing, with close monitoring. As per United States Food and Drug Administration (FDA) label guidelines, low and intermediate-risk patients can also be managed in the outpatient setting without hospitalization and we suspect future real-world series will demonstrate a higher proportion of low and intermediate-risk patients managed in the clinic during the dose ramp-up period.

Potential reasons for increased TLS events may include difficulty in adhering to the exact dose-ramp up schedule or lack of physician/patient education surrounding importance of suggested prophylaxis, laboratory monitoring, and interventions for all patients. Patients may have had differences in comorbidities, such as impaired renal function, which would have made them ineligible for a venetoclax clinical trial and possibly at increased risk for TLS. Deviations in clinical practice initiation of venetoclax from that recommended in the FDA label, in particular the limited use of CT assessment (64.3\%) to establish TLS risk prior to venetoclax initiation, could have led to risk misclassification. Additionally, while investigators were asked to use the Howard criteria to define TLS, it is possible that this mandate was not strictly followed when capturing data, leading to misclassification bias.

To date, little is known regarding reasons for venetoclax discontinuation in clinical practice. In our study, $28 \%$ of all patients discontinued therapy; $53.8 \%$ of these patients discontinued due to progression of CLL excluding Richter's transformation (RT) and $20.5 \%$ discontinued due to toxicity. As in clinical trials, we found CLL progression to be the most common reason for venetoclax discontinuation. In the phase I study of venetoclax for R/R CLL, the overall discontinuation rate of $56 \%$, with $35 \%$ of discontinuations due to CLL progression (non RT) and 20\% due to toxicity. ${ }^{1}$ In the phase II study of patients treated with prior B-cell receptor signal transduction inhibitors, the discontinuation rate was $49.5 \%$ at 14 months median follow up. CLL progression represented $49 \%$ of these discontinuations and AEs represented $11 \%$ of discontinuations. ${ }^{5}$ In this same series, RT was reported in $5 \%$ of patients who discontinued venetoclax. The median time to CLL progression was 8.4 months, and median time to RT was approximately one year..$^{19}$ Anderson et al. reported that, in a group of heavily pretreated patients, $37 \%$ of patients progressed on venetoclax at a median follow up of 23 months, and that $8.2 \%$ of patients discontinued therapy

Table 3. Response rates.

\begin{tabular}{|c|c|c|c|c|c|c|c|c|c|c|c|}
\hline & $\begin{array}{l}\text { Overall population } \\
\qquad n=129\end{array}$ & $\begin{array}{c}\text { Age } \\
>65 \text { years } \\
n=82\end{array}$ & $\begin{array}{c}\text { Age } \\
<65 \text { years } \\
n=47\end{array}$ & $\begin{array}{c}\text { Del 17p } \\
\text { present } \\
n=56\end{array}$ & $\begin{array}{c}\text { Del } 17 p \\
\text { absent } \\
n=69\end{array}$ & $\begin{array}{c}\text { Prior } \\
\text { ibrutinib } \\
\text { therapy } \\
n=107\end{array}$ & $\begin{array}{c}\text { No prior } \\
\text { lbrutinib } \\
\text { therapy } \\
n=22\end{array}$ & $\begin{array}{c}\text { BTK } \\
\text { mutation } \\
\text { present } \\
n=12\end{array}$ & $\begin{array}{c}\text { BTK } \\
\text { mutation } \\
\text { absent } \\
n=22\end{array}$ & $\begin{array}{c}\text { PLCy2 } \\
\text { mutation } \\
\text { present } \\
n=4\end{array}$ & $\begin{array}{c}\text { PLCy2 } \\
\text { mutation } \\
\text { absent } \\
n=28\end{array}$ \\
\hline ORR & $72.1 \%$ & $74.3 \%$ & $68 \%$ & $71.4 \%$ & $72 \%$ & $69.1 \%$ & $86.2 \%$ & $91.6 \%$ & $72.6 \%$ & $75.0 \%$ & $78.6 \%$ \\
\hline CR & $19.4 \%$ & $18.3 \%$ & $21.2 \%$ & $25.0 \%$ & $16.0 \%$ & $17.7 \%$ & $27.2 \%$ & $8.3 \%$ & $18.1 \%$ & $0.0 \%$ & $14.3 \%$ \\
\hline $\mathrm{PR}$ & $52.7 \%$ & $56.0 \%$ & $46.8 \%$ & $46.4 \%$ & $56.5 \%$ & $51.4 \%$ & $59.0 \%$ & $83.3 \%$ & $54.5 \%$ & $75.0 \%$ & $64.3 \%$ \\
\hline SD & $17.8 \%$ & $17.1 \%$ & $19.1 \%$ & $16.1 \%$ & $18.8 \%$ & $19.6 \%$ & $9.0 \%$ & $8.3 \%$ & $18.1 \%$ & $25.0 \%$ & $14.3 \%$ \\
\hline PD & $10.1 \%$ & $8.5 \%$ & $12.7 \%$ & $12.5 \%$ & $8.7 \%$ & $11.2 \%$ & $4.5 \%$ & $0 \%$ & $9.0 \%$ & $0 \%$ & $7.1 \%$ \\
\hline
\end{tabular}

CR: complete response; ORR: overall response rate; PD: progressive disease; PR: partial response; SD: stable disease. 
Table 4. First treatment following venetoclax discontinuation and treatment outcomes.

\begin{tabular}{lcc}
\hline IIreatment & $\begin{array}{c}\text { Number treated } \\
\text { with agent (Percentage of 24 patients } \\
\text { Who received subsequent line of therapy) }\end{array}$ & $\begin{array}{c}\text { Patient level responses (n) } \\
\text { Ibrutinib-based }\end{array}$ \\
Idelalisib-based & $5(20.8 \%)$ & PR (1), SD (2), PD (2) \\
Rituximab monotherapy & $2(8.3 \%)$ & CR (1), No response assessment (1) \\
CAR-T & $3(12.5 \%)$ & PR (2), PD (1) \\
\hline Anthracycline-based (R-CHOP/R-EPOCH) & $2(8.3 \%)$ & PD (2), no response assessment (1) \\
Allogeneic SCT & $3(12.5 \%)$ & CR (2), no response assessment (1) \\
\hline Other & $3(12.5 \%)$ & PR (1), SD (1), PD (2), no response assessment (2) \\
\hline
\end{tabular}

due to other reasons with no patients discontinuing due to toxicity. ${ }^{2}$ We also note these results differ from recent real world BCR inhibitor series where AEs were the most common reason for drug discontinuation, followed by CLL progression. ${ }^{20}$

Even less is known about sequencing of therapies following venetoclax discontinuation. Anderson et al. report outcomes on 25 patients who progressed following venetoclax, 8 with progressive CLL and 17 with RT. In this series, 6 CLL patients with progression, all of whom were ibrutinib naive, were treated with a BTK inhibitor as the first therapy after discontinuation. Five of six (83\%) initially achieved a partial response.

Our study is unique in that we report patient level treatment data on 24 CLL patients progression following venetoclax, which represents the largest series reported to date. These patients are representative of the U.S. population currently treated with venetoclax in that they are treated in the R/R setting, and $89 \%$ had been exposed to a BCR inhibitor prior to venetoclax treatment, most commonly ibrutinib. We found that, following progression on venetoclax, ibrutinib was most commonly selected agent, accounting for $20.8 \%$ of the cases. However, idelalisibbased, rituximab monotherapy, CAR-T, anthracycline based therapy, and allogeneic stem cell transplant were also selected as next therapy in other cases. Interestingly, 3 patients underwent allogeneic stem cell transplant as first therapy after venetoclax. Two achieved a CR and 1 did not have an available response assessment. One patient received an allogeneic SCT as second therapy following venetoclax and achieved CR. Although interpretation of the SCT results are subject to selection bias, they suggest that there is a potential role for effective cellular therapies and should be explored. Our data demonstrate that no clear consensus exists for therapy selection following venetoclax failure and highlights the importance addressing sequencing strategies in future clinical trials. This will become increasingly important as more patients in practice are treated with venetoclax alone or in combination with antibodies and/or ibrutinib.

Our study has several limitations. Data were collected retrospectively by multiple physicians and are subject to differences in clinical experience, practice style, and inconsistencies in chart review. Missing data varied with individual data points and were infrequent. To address this, we included absolute numbers and percentages to highlight any data that was not reported for individual data points. Additional data, including performance status, could offer additional insight but was not collected. Variables and out- comes, including TLS risk categorization, TLS events, and response were documented per physician assessment. Although we recommended the use of iwCLL response criteria, Howard criteria for TLS, and tumor burden classification per package insert, central review of outcomes was outside the scope of this study and, therefore, outcomes may have been subject to misclassification bias. While the case report form captured information on TLS prophylaxis and events, it was not designed to discern the detailed information that would be required to understand rate of TLS by management strategy. This was beyond the scope of this study but future research should consider examining this important information. Additionally, AE assessment was not comprehensive and included data on select AEs such as TLS, hematologic, infection or gastrointestinal toxicities. Applying CTCAE criteria retrospectively may result in underreporting of events and caution is emphasized in interpreting these findings. Indications for treatment were based on treating physicians' discretion and were not specified. Detailed information regarding reason for discontinuation of line of therapy prior to venetoclax was not captured. As we know from prior studies, outcomes can differ significantly in patients who discontinue a kinase inhibitor due to toxicity as compared to progression. ${ }^{8}$ Future studies of venetoclax should consider stratifying patients by these subgroups. While we did include data from community practices, the fact that most patients were treated in academic centers could introduce a selection bias. Our median follow up of 7 months is short and does not capture progressions on venetoclax that occur later.

Despite its inherent limitations, this series represents the largest real-world cohort of CLL patients treated with venetoclax. Neutropenia and thrombocytopenia were the most common toxicities, and progression was the leading cause of venetoclax discontinuation. Venetoclax was active in patients with mutations known to confer ibrutinib resistance as well as in patients with other poor risk features. However, TP53 interruption was associated with an inferior PFS. While we report the largest series of postvenetoclax outcomes, we demonstrate no clear sequencing pattern. Because the number of patients who discontinue venetoclax due to disease progression or toxicity within the first 2 years of initiating therapy is not trivial, understanding how these patients should be subsequently treated is a critical area of future research.

\section{Acknowledgments}

The authors would like to thank Joseph and Cindy Riggs for their support. 


\section{References}

1. Roberts AW, Davids MS, Pagel JM, Kahl BS, Puvvada SD, Gerecitano JF, et al. Targeting BCL2 with Venetoclax in Relapsed Chronic Lymphocytic Leukemia. N Engl J Med. 2016;374(4):311-22.

2. Anderson M, Tam C, Lew T, Juneja S, Juneja M, Westerman D, et al. Clinicopathological features and outcomes of progression of CLL on the BCL2 inhibitor venetoclax. Blood 2017; 129(25):3362-70.

3. Stilgenbauer S, Eichhorst B, Schetelig J, Coutre S, Seymour J, Munir T, et al. Venetoclax in relapsed or refractory chronic lymphocytic leukaemia with $17 p$ deletion: a multicentre, open-label, phase 2 study. Lancet Oncol. 2016;17(6):768-78

4. Coutre S, Choi M, Furman RR, Eradat $H$, Heffner L, Jones JA, et al. Venetoclax for patients with chronic lymphocytic leukemia who progressed during or after idelalisib therapy. Blood. 2018; 12:131(15):1704-11.

5. Jones J, Mato A, Wierda W, Davids M, Choi $\mathrm{M}$, Cheson B, et al. Venetoclax for chronic lymphocytic leukaemia progressing after ibrutinib: an interim analysis of a multicentre, open-label, phase 2 trial. Lancet Oncol. 2018;19(1):65-75.

6. Seymour JF, Kipps TJ, Eichhorst B, Hillmen $\mathrm{P}$, D'Rozario J, Assouline $\mathrm{S}$, et al. Venetoclax-Rituximab in Relapsed or Refractory Chronic Lymphocytic Leukemia. N Engl J Med. 2018; 378(12):1107-20
7. Mato A, Nabhan C, Barr P, Ujiani C, Hill B, Lamanna $\mathrm{N}$, et al. Outcomes of CLL patients treated with sequential kinase inhibitor therapy: a real world experience. Blood. 2016;128(18):2199-205.

8. Mato AR, Hill BT, Lamanna N, Barr PM Ujjani CS, Brander DM, et al. Optimal sequencing of ibrutinib, idelalisib, and venetoclax in chronic lymphocytic leukemia: results from a multicenter study of 683 patients. Annals of oncology : official journal of the European Society for Medical Oncology. 2017;28(5):1050-6.

9. Forum UC. Ibrutinib for relapsed/refractory chronic lymphocytic leukemia: a UK and Ireland analysis of outcomes in 315 patients. Haematologica. 2016; 101(12): 1563-72.

10. Mato AR, Allan JN, Pagel JM, Brander DM, Hill BT, Cheson BD, et al. Front-Lin Ibrutinib Therapy for Chronic Lymphocytic Leukemia (CLL) in the Rea World: Responses, Toxicity, Outcomes and Subsequent Therapies. Blood 2017; 130(Supp 1):3011.

11. Barrientos JC, Kaur M, Mark A, Chung J Driscoll N, Bender A, et al. Outcomes of Patients with Chronic Lymphocytic Leukemia (CLL) after Idelalisib Therapy Discontinuation. Blood. 2015;126(23): 4155.

12. Hallek M, Cheson B, Catovsky D, CaligarisCappio F, Dighiero G, Döhner $\mathrm{H}$, et al Guidelines for the diagnosis and treatment of chronic lymphocytic leukemia: a report from the International Workshop on Chronic Lymphocytic Leukemia updating the National Cancer Institute-Working Group
1996 guidelines. 2008;111(12):5445-56

13. FDA. Highlights of Prescribing Information: Venclexta. 2018.

14. Howard S, Jones D, Pui C. The tumor lysis syndrome. N Engl J Med. 2011; 364(19):1844-54

15. Bland J, Altman D. Survival probabilities (the Kaplan-Meier method). BMJ. 1998; 317(7172):1572

16. Matthews D, Farewell V. 7 The Log-Rank or Mantel-Haenszel Test for the Comparison of Survival Curves In: Basel S Karger A, eds. Using and Understanding Medical Statistics 2007:67-75.

17. Anderson P, Gill R. Cox's regression mode for counting processes: a large sample study. Ann Statist. 1982;10(4):1100-20.

18. Kipps TJ, Fraser G, Coutre SE, Brown JR Barrientos JC, Barr PM, et al. Integrated analysis: outcomes of ibrutinib-treated patients with chronic lymphocytic leukemia/small lymphocytic leukemia (CLL?SLL) with high-risk Prognostic Factors. Hematological Oncology. 2017; 35(S2):109-11.

19. Mato A, Wierda W, Davids M, Cheson B, Coutre S, Choi M, et al. Analysis of PET CT to Identify Richter's Transformation in 167 Patients with Disease Progression Following Kinase Inhibitor Therapy. ASH Abstract 817;2017.

20. Mato A, Nabhan C, Thompson M Lamanna N, Brander D, Hill B, et al. Toxicities and outcomes of 616 ibrutinibtreated patients in the United States: a realworld analysis. Haematologica. 2018; 103(5):874-9. 